\title{
Distributed Middleware Enforcement of Event Flow Security Policy
}

\author{
Matteo Migliavacca ${ }^{1}$, Ioannis Papagiannis ${ }^{1}$, David M. Eyers ${ }^{2}$, Brian Shand ${ }^{3}$, \\ Jean Bacon ${ }^{2}$, and Peter Pietzuch ${ }^{1}$ \\ 1 Imperial College London \\ \{migliava,ip108,prp\}@doc.ic.ac.uk \\ ${ }^{2}$ University of Cambridge \\ \{firstname.lastname\}@cl.cam.ac.uk \\ 3 CBCU/ECRIC, National Health Service \\ brian.shand@cbcu.nhs.uk
}

\begin{abstract}
Distributed, event-driven applications that process sensitive user data and involve multiple organisational domains must comply with complex security requirements. Ideally, developers want to express security policy for such applications in data-centric terms, controlling the flow of information throughout the system. Current middleware does not support the specification of such end-to-end security policy and lacks uniform mechanisms for enforcement.

We describe DEFCON-POLICY, a middleware that enforces security policy in multi-domain, event-driven applications. Event flow policy is expressed in a high-level language that specifies permitted flows between distributed software components. The middleware limits the interaction of components based on the policy and the data that components have observed. It achieves this by labelling data and assigning privileges to components. We evaluate DEFCON-POLICY in a realistic medical scenario and demonstrate that it can provide global security guarantees without burdening application developers.
\end{abstract}

Keywords: multi-domain distributed applications, security policy, information flow control, event-based middleware.

\section{Introduction}

Distributed systems that span multiple organisational or administrative domains are increasingly common in many areas, yet the associated security challenges remain largely unsolved. In the public sector from healthcare to public security, the integration of separate agencies and departments into federations enables the free flow of information and promises better services for citizens. Global companies are moving away from monolithic organisations towards a more dynamic business ecosystem that is reflected in the distributed nature of their software infrastructures. To achieve the necessary degree of integration between software components in such applications, they are often implemented as event-driven architectures [1, in which components, potentially belonging to different domains, process and exchange data in the form of event messages. 
Multi-domain, event-driven applications process and exchange personal, often sensitive, data belonging to different users. As a result, organisations have to abide by information handling policies, frequently stemming from data protection laws. Such policies often refer to the flow of sensitive data within the system. For example, the Department of Health in the UK stipulates that any access to a patient's electronic health record must be controlled by strict protocols 11 An open problem is how to encode and enforce such flow-based security policies in the context of multi-domain event-driven applications. In particular, there is an impedance mismatch between high-level policies governing the handling of confidential data and low-level technical enforcement mechanisms.

Enforcing security policies in multi-domain, event-driven applications is challenging for several reasons: (1) due to the complexity and scale of applications, there is a risk that software faults may render policy checks ineffective. A single component that omits an access control check may reveal a confidential patient record to the outside world; (2) the integration of third-party components and libraries, often without source code auditing, may mean that necessary policy checks are omitted altogether. For example, a third-party developer may have a different interpretation of a security policy and decide incorrectly that revealing a patient record to an insurance provider is acceptable; (3) application deployments across multiple administrative domains introduce issues of trust and legal responsibility when enforcing security policies. For example, a hospital domain may trust an insurance provider with billing-related data but not with a complete patient health history. All these factors put the managed data at risksecurity violations of organisations' private data may be disastrous; violations of third-party user data may make them liable to lawsuits.

Traditional event-based and message-oriented middleward2 leaves the task of enforcing security policy to application developers. They have to include appropriate policy enforcement points (PEPs) in application components that carry out checks before executing sensitive operations. The middleware may provide support for the implementation of PEPs in the form of access control models such as access control lists and capabilities. However, these are low-level mechanisms that require the configuration of permissions at the granularity of individual operations. This makes it hard to realise a high-level security policy correctly, in particular, in distributed, multi-domain environments with different levels of trust between organisations.

To address the above security concerns, we argue that it should be possible to express high-level security policy as constraints on permitted data flows throughout a distributed, event-driven application. It should be the responsibility of the middleware to enforce such security policy uniformly across domains by taking a data-centric view - policy enforcement should occur automatically when data flows cross boundaries between components and domains, instead of carrying out access control checks over individual operations.

\footnotetext{
${ }^{1}$ See http://www.nigb.nhs.uk/guarantee/2009-nhs-crg.pdf

${ }^{2}$ For example, IBM's WebSphere MQ product: http://www.ibm.com/webspheremq
} 
In this paper, we describe DEFCON-POLICY, an event-based middleware that enforces event flow security policy in distributed, multi-domain applications. Flow policy is expressed in the DEFCon Policy Language (DPL) as high-level constraints on permitted data flows between components. The middleware takes a DPL specification and translates it into an equivalent assignment of security labels, which are associated with data flows, and privileges, which enable components to send and receive labelled data. To prevent event flows that would violate the security policy, DEFCON-POLICY sandboxes components after they have received sensitive data controlled by the policy.

This approach makes policy enforcement transparent to application developers and does not add complexity to the application implementation. We evaluate DEFCON-POLICY with a case study of a healthcare scenario with multiple distributed domains. Our results show that DEFCON-POLICY can realise a complex, realistic security policy and enforce it with an acceptable runtime overhead.

In summary, the paper makes the following main contributions:

- a high-level event flow policy language for expressing constraints on distributed event flows in multi-domain, event-driven applications;

- the design and implementation of a middleware that can enforce event flow policy by monitoring event flows between application components and restricting them to ensure policy compliance;

- the evaluation of this approach as applied to security policy enforcement in the context of a realistic medical scenario.

The rest of the paper is organised as follows. In 92 we explore security problems in multi-domain, event-driven applications. We present DPL, our event flow policy language, in \$3. In 94 , we describe how the DEFCON-POLICY middleware enforces event flow policy in a distributed setting by controlling the flow of events between distributed components. Evaluation results are presented in $\$ 5$, The paper finishes with related work (\$6) and future work and conclusions (\$7).

\section{Security in Multi-domain, Event-Driven Applications}

In this section, we describe the problem of providing security guarantees in distributed systems that involve multiple organisational domains. We introduce a sample healthcare workflow and show how event-based middleware can naturally support it but struggles to cover security challenges. Based on this analysis, we propose to enforce high-level security policy by the middleware.

Figure 1 gives an example of a data processing workflow in a healthcare scenario with multiple domains. It is representative of the types of workflows found in the UK National Health Service (NHS). The figure shows domains (dashed regions) and software components within those domains. In this scenario, a patient is worried about a lump on his arm, and visits his general practitioner (GP) for a consultation. The GP takes a skin biopsy, which she sends, together with an electronic biopsy request containing information about the patient, to an NHS pathology laboratory for testing (edge $\mathrm{e}_{1}$ ). There, a pathologist analyses the 


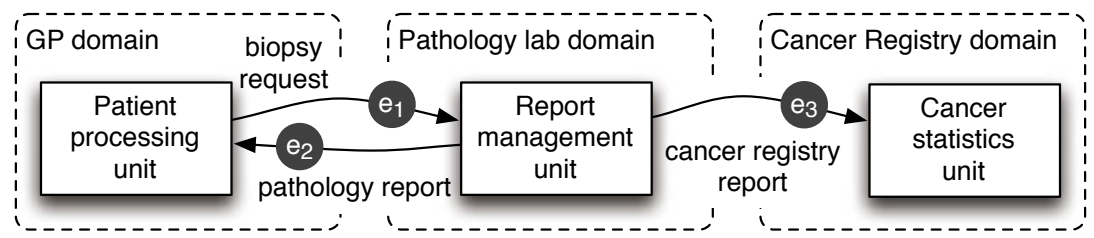

Fig. 1. Multi-domain healthcare scenario. Events are exchanged between processing units belonging to multiple domains.

sample and produces an electronic pathology report. The lab sends this pathology report back to the GP (edge $e_{2}$ ), and also sends a copy to the regional cancer registry (edge $e_{3}$ ), but only if there is any evidence of cancer.

\section{$2.1 \quad$ Event-Based Middleware}

A software system that supports the above workflow can be effectively realised as an event-driven architecture [1]. In this architectural model, data is presented as structured event messages or events. The software components of an application are implemented as a set of event processing units that receive, process and emit events. Events are usually transformed by a sequence of units. Units are hosted on physical machines and belong to a given organisational or administrative domain. An event-based middleware dispatches events between units, either locally or involving network communication between machines.

In the above scenario, the GP, the pathology lab and the cancer registry each form a domain. There are three types of events flowing between them: the biopsy request event $e_{1}$, the pathology report event $e_{2}$ and the cancer registry report event $e_{3}$. These events are exchanged between units belonging to the different domains: a patient processing unit in the GP domain, a report management unit in the pathology lab domain and a cancer statistics unit in the cancer registry.

\subsection{Information Security}

The above workflow has events that contain confidential patient data, which makes information security important. Their propagation between the different parts of the system is regulated by corresponding data protection legislation 3 It states that the following security guarantees must be maintained at all times:

1. Pathology reports may be sent only to the requesting GP or a cancer registry.

2. Cancer registries may only receive cancer-related pathology reports.

3. Only doctors in the pathology lab may view sensitive patient data.

In general, the security goals in such scenarios are to prevent leakage of data to unauthorised units or third-parties and to ensure the integrity of data that

\footnotetext{
${ }^{3}$ http://www.nhs.uk/choiceintheNHS/Rightsandpledges/NHSConstitution/ Documents/COI_NHSConstitutionWEB2010.pdf
} 
units use for input. For example, the pathology report event should not be sent to any other units outside of the GP and Cancer Registry domains. In addition, the GP domain should only accept genuine pathology reports as input.

In terms of a threat model, information security can be violated in a number of ways: software bugs in the implementation of units can leak sensitive events or make units accept bogus input data; malicious developers can include back-doors in unit implementations to obtain unauthorised access to data; and units operating in different domains may handle security policy differently due to inconsistent interpretations at implementation time. All of these problems are enabled by the fact that security concerns are distributed across the implementation of many units and are disconnected from the global security requirements. Any unit in the system can potentially violate information security.

Security policy. To ensure that a multi-domain, event-based application guarantees information security, we argue that a policy administrator should first express security concerns in a high-level policy language. By separating security policy from unit implementations, the policy administrator can focus on the high-level security goals of a multi-domain application, without being overwhelmed by implementation details.

A key observation when expressing security policy is that the required security guarantees, as the ones described above, usually pertain to the event data and, more specifically, focus on the flow of events through the system. An event flow security policy should therefore control the propagation of event flows through the system. This is in contrast to fine-grained security policy found in access control systems that usually governs permitted operations. For example, when the biopsy request event is received by the report management unit, it is trusted to manage the data appropriately. Any operations carried out by the report management unit internally do not need to be checked, as long as interactions of the unit with other units are controlled.

Policy enforcement. Current implementations of multi-domain, event-driven applications leave the overall enforcement of security policy to the developers of units. A frequent approach is to introduce an access control layer around units, which carries out ad hoc policy checks at the input and output of events. This is not only error-prone but also makes it challenging to enforce security properties that rely on the behaviour of a sequence of event processing units.

In contrast, we want to enforce event flow policy by the middleware itself, independently of the implementation of processing units. For this, the middleware must track the flow of events between components in order to provide end-to-end security guarantees that do not depend on the correct implementation of each individual unit. This assumes that the middleware implementation is correct and can be trusted to enforce event flow policy. In practice, this is a reasonable assumption because only a small part of the middleware implementation is involved with policy enforcement.

Information flow control. Since event flow policy expresses limitations on the flows of events throughout the system, the middleware must be able to prevent invalid flows. This idea of information flow control has been successfully 
applied in different domains for achieving security guarantees, including operating systems [23], programming languages [4], web applications [56] and highperformance event processing [7].

In previous work, we proposed a Decentralised Event Flow Control (DEFC) model [7] for controlling the flow of events in event-driven applications. DEFC associates events with labels, that "contaminate" units that receive them: data output by a contaminated unit must include the labels of the events that contaminated it. As detailed in $\$ 4.2$, this mechanism is key for implementing mandatory tracking of security properties for data processed in the system. Units may bypass constraints imposed by labels associated with events only when they possess privileges over them. As we show in 4 , the DEFC model provides an appropriate low-level enforcement mechanism for event flow policy. However, it must be extended to support multiple domains that may enforce policies differently.

\section{Event Flow Policy}

In this section, we introduce the DEFCon Policy Language (DPL), our language for event flow policy specification. Based on the previous analysis of security in multi-domain, event-based applications, the design of DPL aims to satisfy the following set of requirements:

- Security policies should take a data-centric view, providing end-to-end guarantees for confidentiality and integrity of event flows in the system.

- Security policies should be independent of the functional implementation of processing units and be supported across legacy processing units.

- Security policies should be separate from the details of the enforcement mechanism at the middleware level.

- Security policies should be enforceable efficiently, without resulting in an unacceptable degradation of event processing performance.

We describe DPL with reference to a financial scenario, as illustrated in Figure2, Within the processing system of a bank, several functions exist: from investment activities to accounting on behalf of clients to internal risk assessment. Flows of

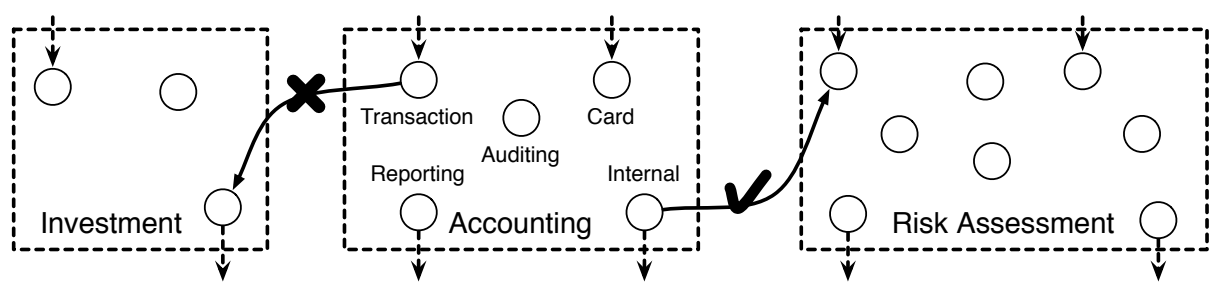

Fig. 2. Protection of flow categories within a bank. Flow categories define boundaries around data and specify which units can pass data over those boundaries. Transaction, as an input unit, cannot send data to the investment flow, while Internal, an output unit, can extract data from the flow. 
information are exchanged within each function and among different functions. We consider the case of a bank that wants to improve the security of the software component that processes customer account information. The goal is to ensure that account information cannot be corrupted or leaked by software faults or malicious behaviour of components.

The events in this scenario can be divided into event flow categories. An event flow category, such as accounting flow, is used to identify events with distinct security requirements, for example, by pertaining to data containing customers' account details. Alternatively, a flow category could group all data belonging to a single user.

\subsection{Event Flow Constraints}

Our event flow policy provides security guarantees through the definition of event flow constraints on flow categories. We focus on two ways that policy specification can distinguish flows of information by applying flow constraints and we name them vertical and horizontal flow separation. Vertical separation relates to flow constraints that should hold across the end-to-end processing of events, from input to output. Horizontal separation is used to isolate the processing at one stage from the processing being done in another, and is typically used to achieve security guarantees related to functional transformations such as data cleaning, auditing and anonymisation.

In DPL, event flows constraints have the following syntax:

$$
\begin{aligned}
& \text { flow_constraint }::=\langle\text { flow_name }\rangle \text { ' }: \text { ' ' }\{\text { ' flow_part (', ' flow_part)* ' }\} \text { ' ' . ' }
\end{aligned}
$$

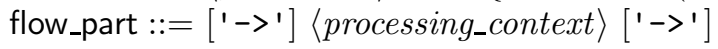

As an example, the following DPL specification encodes the flow constraints in the above banking scenario:

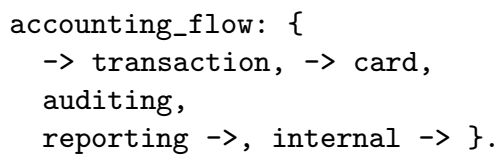

All flow constraints must name an event flow category (i.e. accounting_flow above), and state whether the flow parts (i.e. card, transaction, etc.) can receive or produce events within the flow. Flow parts indicate the processing context, which can be a unit that sees and alters the event flow 4 The inclusion of a unit as a flow part, without any further annotation, means that the unit is sandboxed within the context of the specified event flow. In other words, such units are isolated so that they can only input and output events from and to other units within that event flow.

Parts of event flows contain annotations to indicate that they are able to cause events to flow in or out of the event flow. Input units, preceded by a $\rightarrow$ prefix, such as transaction and card are constrained to output events only within the

${ }^{4}$ To simplify discussion, we assume for now that a processing context is a single unit; we relax this assumption in 3.2 , when we address the general case. 


\subsection{Abstracting Processing Context}

So far, we illustrated the use of DPL in small-scale contexts. We assumed that policy administrators had global knowledge of all processing units that participate in event flows. In such scenarios, it is possible to link policy fragments in the form of flow constraints directly to the units that are constrained by these fragments. However, such an approach is not feasible in larger deployments where the policy spans multiple domains and no domain has control over the details of event processing in other domains. For example in our accounting scenario, including units from a third party would tie in the policy with the units' design: changes to the design would require changes to the event flow specification.

To apply event flow control in a large distributed setting, it is therefore necessary to abstract the relationship between flow policies and units. We achieve this by introducing hierarchical names that correspond to event processing contexts. The hierarchical nature of processing contexts facilitates support for multidomain use of event flow policy: we can map the organisational structure of domains to the hierarchical structure of processing contexts. Also, by using a federated naming service analogous to the domain name system (DNS), the control over subcontexts can be delegated to the domains themselves.

Processing context names provide a common, consistent naming structure to correlate processing units and policies belonging to different organisations. Flow constraints can refer to processing context names, which then map to actual event processing units. This relaxes the previous assumption in $\$ 3.1$ that each unit maps to exactly one processing context. When a flow constraint states a processing context, the constraint applies to all units that are directly part of the context and to all units that are part of any sub-contexts.

We illustrate processing contexts with two examples. As the first example, we refine the flows that are internal to the previously introduced reporting context from Figure 2 by specifying the following flow constraint:

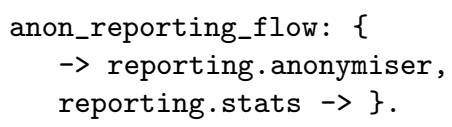

This flow names two sub-contexts of reporting: an anonymiser and stats. The stats context is reachable only through the anonymiser. All units assigned to reporting.stats can only receive data from reporting. anonymiser, while units in anonymiser or directly in reporting are still constrained by any flow constraint mentioning the reporting context. As a multi-domain example, we can consider the following version of the accounting flow:

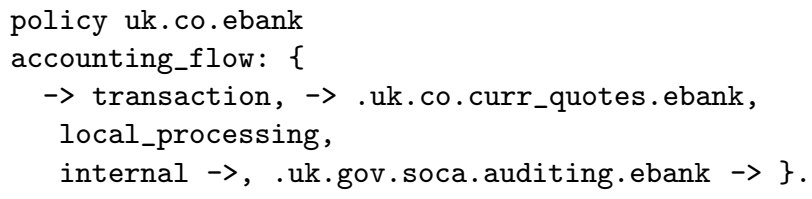

In this policy, processing contexts not starting with a dot are treated as relative to the domain specified in the policy header, while fully-qualified names can 


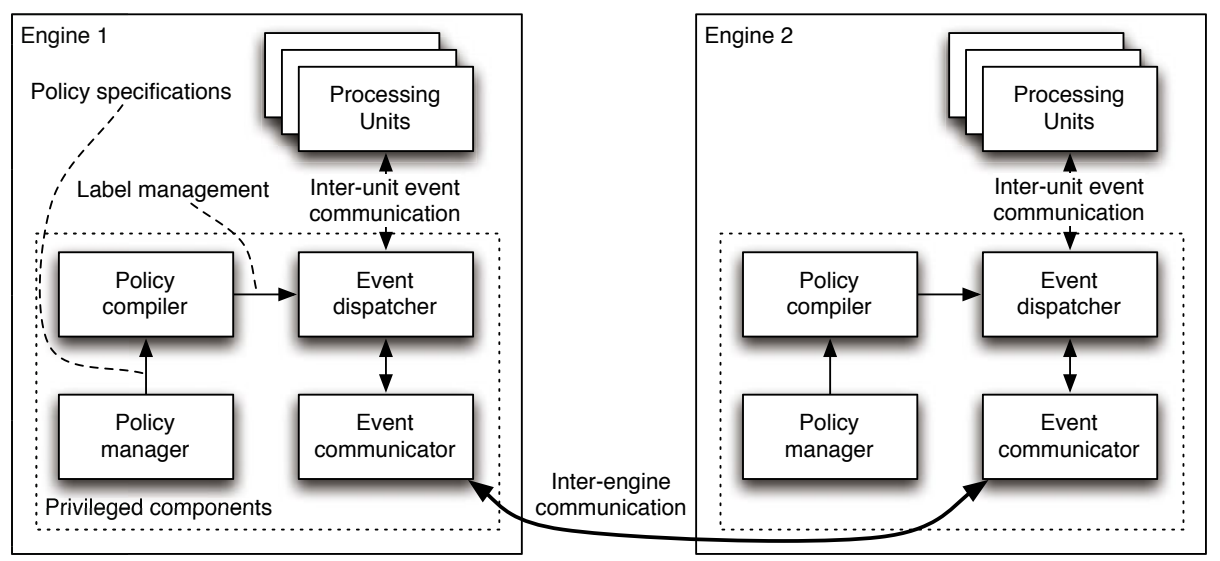

Fig. 4. DEFCon-Policy architecture. Multiple engines house processing units that communicate using message passing while information flow is tracked using labels.

refer to arbitrary contexts. This example has an external provider of quotes for foreign currencies and the UK Serious Organised Crime Agency (SOCA) as an output context. The two organisations with control over these processing context names define processing units operating in these domains, and authorise foreign organisation (ebank in this case) to define policies relating to these contexts.

\section{Distributed Event Flow Middleware}

Given a set of policies described in DPL, we enforce them using the DEFCoNPOLICY middleware. It implements distributed DPL policies by translating them into local communication constraints on processing contexts and enforces them as units execute. The architecture of the DEFCON-POLICY middleware is shown in Figure 4 and consists of the following parts:

Engines. Engines are responsible for policy enforcement in one or more processing contexts. Each engine hosts processing units and isolates them from each other to be able to sandbox units. Engines also control communication channels to and from other engines and outside systems. Engines manage the internal flow of events using the DEFC security model (cf. \$4.2).

Event dispatcher. The event dispatcher supports asynchronous communication between units in the form of publish/subscribe communication. Publish/subscribe allows units to express their interest in events that match a subscription filter. Events are dispatched in compliance with security labels.

Policy manager. As part of each engine, a policy manager is responsible for locally checking and authorising DPL policies constraining processing contexts local to that engine. In a small-scale deployment, policies can be checked and deployed manually; in more complex deployments, the policy managers of different engines coordinate to set-up policies (cf. 4.1). 
Policy compiler. The policy compiler translates DPL policies into security labels and privileges in the DEFC model used for enforcement (cf. 4.1).

Event communicator. In each engine, the event communicator is responsible for securely propagating protected events between engines. It guarantees that events are labelled correctly in each engine trusted by the policy when they are exported and imported over the network (cf. \$4.2).

\subsection{Distributed Policy Management}

To support large multi-domain deployments, DEFCON-POLICY needs to handle many processing contexts deployed in many engines. In such a scenario, policy set-up and management needs support from the middleware. To set up a policy, before enforcement can begin, DEFCON-POLICY performs a series of steps:

1. Context to engine resolution. After a new DPL policy has been submitted, the DEFCON-POLICY middleware first resolves engines responsible for processing contexts mentioned in the policy, thus locating the deployed units to constrain. The resolution from processing contexts to engines is performed through a distributed directory system. Such a directory service can be federated so that each organisation owns a part of the namespace and can delegate subparts to other organisations.

2. Engine trust verification. Engines have to verify that remote engines involved in a policy can be trusted to enforce event flow constraints defined in the policy. This is important because remote engines may belong to independent administrative domains. For example uk.co.ebank.transactions from $\$ 3.2 \mathrm{might}$ map to a local engine defcon.ebank.co.uk, while uk.co.curr_quotes.ebank might map to engine defcon. curr_quotes.co.uk externally hosted.

In the most general case, each domain, such as ebank, can specify the set of DEFCON-POLICY engines that it trusts for enforcement of its policies. These can be specified per organisation, per policy or per flow. We assume that units are deployed on engines with sufficient trust, such as the local engine, to support their execution. Referring to processing contexts by a fully-qualified name is an assertion of trust in the remote policy enforcement of that domain.

3. Policy deployment and authorisation. Once engines are verified, the policy is deployed on all relevant engines. The policy managers on each engine check if the deployed policy is authorised with respect to the contexts involved. Such authorisation may be implemented by using PKI infrastructurf for example. Digitally signed policies, and information about the signing certificates, can be integrated with the directory service exploited in step 1.

4. Policy checking. Before a policy is enforced, DEFCON-POLICY checks that the new policy is not inconsistent (cf. vertical separation example in \$3). An inconsistent policy may violate liveness properties by leading to units that are unable to receive or send any events because of policy constraints.

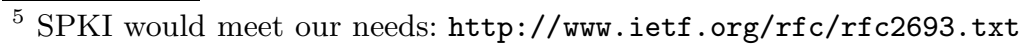




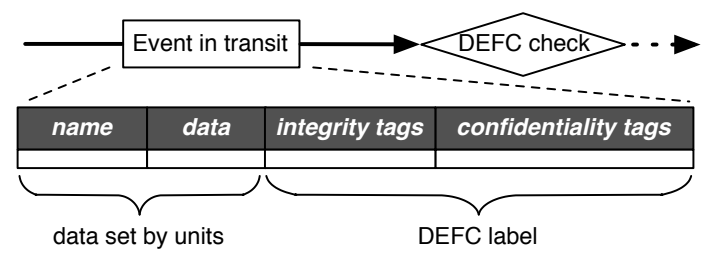

Fig. 5. An event with a DEFC label. DEFC labels are not controlled by units, but are used to enforce event flows.

To check the policy, the policy manager recursively retrieves policies related to the processing contexts specified in the new policy. It then performs a graph traversal to check if, for all units, there exists at least one event flow path from the external world to their input (reachability) and at least one path from their output to the external world (observability).

This policy checking algorithm can be formalised as follows. Let $P$ be the set of all possible processing contexts. The goal of the algorithm is to check the compatibility of a set of flow constraints $F$ where $F \subseteq 2^{P} \times 2^{P} \times 2^{P}$. We can first compute whether two units can send an event to each other according to $F$ :

$$
\begin{aligned}
\operatorname{canSendTo}(x, y) \Leftrightarrow & \Leftrightarrow\left(F_{\text {in }}, F_{\text {sand }}, F_{\text {out }}\right) \in F: F_{\text {all }}=F_{\text {in }} \cup F_{\text {sand }} \cup F_{\text {out }} \\
& \left(x \in\left(F_{\text {in }} \cup F_{\text {sand }}\right) \Rightarrow y \in\left(F_{\text {sand }} \cup F_{\text {out }}\right)\right) \wedge \\
& \left.\left(x \in F_{\text {out }} \vee x \notin F_{\text {all }}\right) \Rightarrow\left(y \in F_{\text {in }} \vee y \notin F_{\text {all }}\right)\right)
\end{aligned}
$$

and based on that, infer reachability via multiple hops:

$$
\begin{aligned}
\operatorname{canReach}(x, y) \Leftrightarrow & \exists n>0, \forall i \in[1, \ldots, 2 n], z_{i} \in P \\
& \wedge\left(z_{1}=x \wedge z_{2 n}=y\right) \wedge \operatorname{canSendTo}\left(z_{i}, z_{i+1}\right)
\end{aligned}
$$

Then we consider a unit that is external to all flow constraints of $F$, i.e. operates in an unconstrained context, modelling the external world $\phi$. Given $\phi \in P: \forall\left(F_{\text {in }}, F_{\text {sand }}, F_{\text {out }}\right) \in F, \phi \notin F_{\text {in }} \cup F_{\text {sand }} \cup F_{\text {out }}$, the following definitions hold:

$$
\begin{aligned}
\text { isObservable }(x) & \Leftrightarrow \operatorname{canReach}(x, \phi) \\
\text { isReachable }(x) & \Leftrightarrow \operatorname{canReach}(\phi, x)
\end{aligned}
$$

The check succeeds if and only if, given $F$,

$$
\forall x \in P \text { : isObservable }(x) \wedge \text { isReachable }(x)
$$

at which point the policy is ready for enforcement.

\subsection{Enforcement of Event Flow Constraints}

After policies have been distributed to engines, the DEFCON-POLICY middleware sets up runtime enforcement within an engine and between engines.

DEFC model. Event flow constraints specified in DPL are enforced at runtime according to the Decentralised Event Flow Control (DEFC) model [7]. In this 
model, as shown in Figure 5, events are structured messages that consist of (1) a named data part that units can manipulate and (2) a DEFC label. The data part contains the payload of the event whereas the DEFC label restricts its flow 6

A DEFC label $(S, I)$ is composed of a confidentiality label $(S)$ and an integrity label $(I)$. Labels are sets of tags, each representing a concern over confidentiality or integrity of the event. A tag is implemented as a unique bit sequence.

Processing units are also assigned a label $L_{p}$ that represents the confidentiality and integrity of information contained in their state. Units can create events or process events that they receive, provided that their label can flow to the labels of the events. Intuitively, a unit cannot read data that is "more confidential" than its label, or write data that has "higher integrity" than itself. More precisely, an event flow is only allowed if the source label $L_{s}$ and the destination labels $L_{d}$ satisfy a partial order can-flow-to relation $\preceq$ :

$$
L_{s}=\left(S_{s}, I_{s}\right) \preceq L_{d}=\left(S_{d}, I_{d}\right) \Longleftrightarrow S_{s} \subseteq S_{d} \wedge I_{s} \supseteq I_{d}
$$

Units possess privileges that allow them to change labels. Adding a tag $t$ to a label requires the $t^{+}$privilege, which for integrity is called an "endorsement privilege" - it endorses the unit's state, allowing it to produce higher integrity data. Removing $t$ from a confidentiality label requires the $t^{-}$privilege, called a "declassification privilege" - it declassifies the unit's state allowing it to produce unclassified data. Privileges held by units also determine if a unit can communicate externally. Only a unit that holds endorsement privileges $t^{+}$for all tags in $I$ and declassification privileges $t^{-}$for all tags in $S$ can freely exchange events with the outside world.

DEFC also controls the delegation of privileges between units. Only if a unit possess the privilege-granting privileges $t_{a u t h}^{+}$and $t_{\text {auth }}^{-}$, it is permitted to delegate its endorsement and declassification privileges $t^{+}$and $t^{-}$to other units.

Policy translation to DEFC. The DEFC model is used to enforce event flow constraints specified in DPL policy specifications. Each flow constraint $f$ is associated with a tag pair $\left(c_{f}, i_{f}\right)$ to protect flow confidentiality and integrity. Tags and privileges are assigned to units in the following way:

(1) Sandboxed and output units have $i_{f}$ in their integrity label, constraining them to receive only events that also contain $i_{f}$. (2) Sandboxed and input units also have $c_{f}$ in their confidentiality label, thus being constrained to have their output contain $c_{f}$. (3) Input units are given $i_{f}^{+}$and therefore can produce events with $i_{f}$, even if they do not have $i_{f}$ in their label. This means that they can receive events form "outside of the flow". (4) Output units are given $c_{f}^{-}$to produce data without $c_{f}$ in their label, even if their confidentiality label contains $c_{f}$ to be able to receive data from the flow. When a unit is mentioned in multiple flow constraints, its label is the conjunction of all listed constraints.

In the multi-domain example from $\$ 3.2$, the policy manager on the engine def con.ebank.co.uk, responsible for the transactions, local_processing and internal subdomains of uk.co.ebank, would create $i, c$ tags to represent the

${ }^{6}$ We ignore multiple data parts here; see [7] for more detail on the DEFC model. 
flow uk.co.ebank. account_flow. The manager would then instantiate the processing unit parts of those contexts with a label $L=(\{c\},\{i\})$, it would also bestow $i^{+}$in transactions, and $c^{-}$in internal, keeping $i_{\text {auth }}^{+}$and $c_{\text {auth }}^{-}$for itself. These privileges can be used by the policy manager in case of a policy change that would require a reconfiguration of privileges.

Inter-engine communication. Tags and privileges that are allocated by policy managers in engines have local meaning. Engines use these to restrict communication between units in the local engine. However, units that are able to process data of a given flow should be able to exchange events even if they are located on different engines. This requires the exchange of events between two engines over the network. It cannot be achieved by just giving units endorsement or declassification privileges because this would enable them to communicate with units outside of the flow, without event flow control.

To address this problem, DEFCON-POLICY provides a trusted proxy unit, called an event communicator. The event communicator is delegated endorsement and declassification privileges for a given event flow and can then transfer events to the event communicator in another engine. As part of this process, the tags associated with events are translated at the receiver's engine by the event communicator to equivalent tags for local enforcement. This mapping between tags on different engines is set up on demand, on the basis of the policy specifications shared between the engines during policy deployment (\$4.1).

We illustrate inter-engine communication in the context of the example above. The policy manager on the engine defcon.curr_quotes.co.uk, which is responsible for the uk.co.curr_quotes.ebank context, can allocate tags $i, c$ to represent the uk.co.ebank.account_flow flow. It initialises units in the context to $L=(\{c\},\{i\})$, granting the $i^{+}$privilege to them. The units from the uk.co.curr_quotes.ebank context cannot communicate directly with units in uk.co.ebank.local_processing, which are sandboxed in the accounting flow.

Each policy manager instantiates an event communicator, granting it the $i^{+}$ and $c^{-}$privileges. When a unit in the uk.co.curr_quotes.ebank context sends an event to uk.co.ebank.local_processing, the event is received by the communicator, which exports it from the accounting flow by exercising its declassification privilege. The event is then transmitted securely, for example, using an encrypted transport-level connection, to the other event communicator. The second event communicator possesses the $i^{+}$privilege, which enables it to insert the received event in the accounting flow in the other engine.

\section{Evaluation}

We evaluate the effectiveness of DPL and DEFCON-POLICY with respect to specifying and enforcing security policy in a multi-domain, event-driven application. We focus on the ease-of-use from a software developer's standpoint and also experimentally evaluate the performance impact of the middleware. 


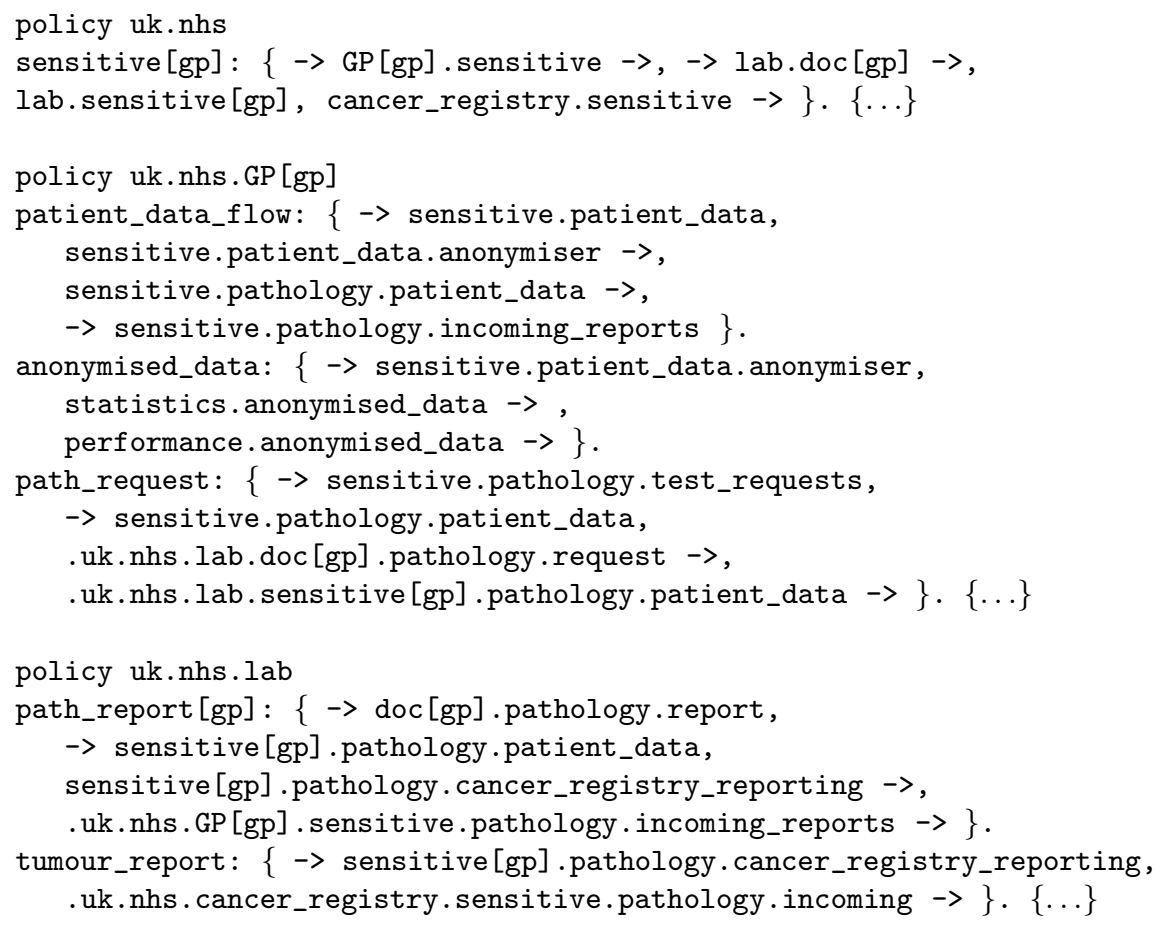

Fig. 6. Extract of the healthcare policy scenario in DPL. Constraints not related to managemet of sensitive medical data are omitted.

\subsection{Healthcare Case Study}

In our case study, we examined an NHS policy involving GPs, Pathology Laboratories, a Primary Care Trust (PCT), the UK Office of National Statistics (ONS) and a Cancer Registry. Figure [6] shows an extract of the policy that enforces the guarantees introduced in 92

An overarching NHS policy (lines 1-3) specifies a high-level constraint that sensitive data are controlled and partitioned by GPs. Partitioning is enforced by the use of a constraint parametrised by gp. Data processing in the laboratory is also partitioned by GP and only doctors within the lab can see and contribute to confidential information (lines 2-3). Finally, Cancer Registries can receive sensitive information for computing statistics about tumours (line 3).

GPs specify their own local policy (lines 5-16) to refine and extend the global policy. In this example, all GPs have the same policy: patient data can be transformed by an anonymiser into anonymised data (line 7), which in turn can be used for computing statistics by the ONS and measuring performance by the PCT (lines 10-12). Alternatively, patient data can be used to generate pathology requests that are to be sent to a lab (lines 8 and 13-16), while reports received from the lab can be combined with patient data (line 9). The lab pathology 
reports can either be sent back to the same GP (line 22) or included in tumour reports (lines $23-24$ ) by specific reporting units (line 21).

Policy enforcement in DEFC. After the policy is specified in DPL, it is compiled into tags that are used for DEFC enforcement. We now show how the resulting tag assignment enforces the guarantees presented in 92 Each flow constraint is enforced by a tag pair, which we represent symbolically as $\left(i_{x}, c_{x}\right)$ where $x$ is the line at which the constraint is defined in Figure 6, Pathology reports are produced within the lab.doc[gp].pathology.report context, specific to each $G P$, that is tainted by $c_{19}^{g p}$. The declassification privilege for this tag, $c_{19}^{g p+}$, is held by the corresponding GP [gp] . sensitive .pathology . incoming_report context and not by any other context under a different GP. The only other context with declassification privilege for the report tag is lab.sensitive [gp] .pathology . cancer_registry_reporting. It is, however, tainted by tag $c_{23}$, which can be removed only by cancer_registry . pathology . incoming. This completes the enforcement of the first guarantee protecting sensitive pathology reports.

As the cancer_registry. pathology . incoming context is tainted by $i_{23}$, lab.sensitive[gp].pathology.cancer_registry_reporting, holding the $i_{23}^{+}$privilege, is the only context that can send data to it. Furthermore, as units in this context drop reports not classified as cancerous, the second guarantee on Cancer Registry input is enforced.

To enforce the third guarantee, the GP[gp].sensitive.patient_data context is protected by $c_{2}^{g p}$ and $c_{6}^{g p}$. Only units under lab.doc[gp].pathology.request can reveal sensitive data to authenticated doctors within the lab because, as a sub-context of GP [gp], they have the $c_{2}^{g p-}$ privilege. While these units do not hold privileges for $c_{6}^{g p}$, units in GP [gp].sensitive.pathology.patient_data can exchange $c_{6}^{g p}$ with $c_{13}^{g p}$ for which units in lab.doc[gp].pathology.request have the $c_{13}^{g p-}$ privilege.

The policy fragment consists of 24 lines. It generates $10 n+2$ tags and distributes $37 n+1$ privileges where $n$ is the total number of GPs in the system. Assuming that one unit is instantiated in every context, at least $14 n+2$ units must be initialised with correct taints. To provide this initial set-up manually, a programmer would have to call the low level DEFC API at least $24 n+4$ times. Instead, these calls, the creation of tags and the distribution of privileges are automatically carried out by DEFCON-POLICY.

\subsection{Performance Overhead}

In this section, we present an experimental evaluation of the performance impact of enforcing event flow policy using DEFCON-POLICY. We measure overhead as a micro-benchmark in terms of (1) the end-to-end event propagation latency between a set of units and (2) the throughput of event processing. For these experiments, we deploy the following simple security policy:

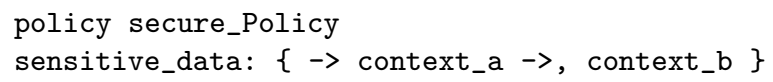


Table 1. Performance overhead of DEFCon-PoLICY middleware

\begin{tabular}{|c|c|c|c|c|c|c|}
\hline \multicolumn{3}{|c|}{$\begin{array}{c}\text { Configuration } \\
\text { Engines TLS Policy }\end{array}$} & $\begin{array}{l}\text { Throughput } \\
\text { (Events/sec) }\end{array}$ & Penalty & $\begin{array}{c}\text { Latency } \\
(\mathrm{ms})\end{array}$ & Penalty \\
\hline 1 & $\mathrm{n} / \mathrm{a}$ & $x$ & 99,723 & - & 0.028 & - \\
\hline 1 & $\mathrm{n} / \mathrm{a}$ & $\checkmark$ & 82,334 & $17.4 \%$ & 0.030 & $7.1 \%$ \\
\hline 2 & $x$ & $x$ & 62,215 & - & 0.268 & - \\
\hline 2 & $\checkmark$ & $x$ & 42,344 & $31.9 \%$ & 0.283 & $5.6 \%$ \\
\hline 2 & $\checkmark$ & $\checkmark$ & 37,500 & $39.7 \%$ & 0.294 & $9.7 \%$ \\
\hline
\end{tabular}

This policy specifies that only units in context_a can cause events to flow in or out of the sensitive_data flow. Units in context_b can perceive and process such events without the ability to disclose them. A single unit $A$ and a single unit $B$ are instantiated in each context, respectively. We compare processing latency and throughput while varying the following parameters:

1. Number of engines. The units/contexts are deployed in a single engine or in two different engines.

2. Network encryption. When network communication is involved, Transport Layer Security (TLS) can be used to encrypt data.

3. Policy enforcement. The engines enforce that events are propagated according to secure_policy.

Our experiments are conducted on two Intel Core 2 Duo E6850 3 GHz machines with a maximum of $1 \mathrm{GiB}$ of heap memory allocated per engine. We use Sun's unmodified JVM 1.6.0.06 on Ubuntu 8.04. The average network round trip-time between the machines is $0.18 \mathrm{~ms}$. Each event contains a single integer.

Table 1 shows the average throughput and the $95^{\text {th }}$ percentile of latency for events sent from unit $A$ to unit $B$ and back to $A$. As this experiment does not involve actual event processing, it mainly stresses the event dispatching mechanism. In the single-engine configurations, DEFCON-POLICY enforcement introduces an overhead of $17.4 \%$ for throughput and $7.1 \%$ for latency. This is the result of storing, propagating and checking tags at runtime.

The overall lower performance achieved in the two-engine configurations is a consequence of the work carried out by the event communicators. Throughput is reduced by $31.9 \%$ due to network encryption. On top of this, DEFCon-PoLICY enforcement introduces a further relative overhead of only $11.4 \%$ for throughput and $3.9 \%$ for latency. We believe that the overhead of policy enforcement becomes even more marginal for realistic applications with more costly processing 7

\section{Related Work}

Middleware. Messaging middleware, and event-based middleware in particular, such as Sun JMS or IBM WebSphere support efficient exchange of information

\footnotetext{
${ }^{7}$ Note that Sun's JVM does not fully enforce unit isolation; the overhead imposed to achieve such isolation was the focus of previous research [7].
} 
in large-scale distributed systems. Security in these systems usually focuses on access control at the boundary of the middleware API rather than end-to-end tracking of information. Any component with access to multiple channels can transfer information between them. As such each component needs to be trusted to comply with integrity and confidentiality requirements of messages.

Policy. Most approaches to policy specification focus on actions (i.e. privileges) rather than data, e.g. access control lists and role-based access control. Higher level firewall policy languages $[8$ facilitate the definition of rules for "allow/deny" actions, but such policies are only enforced locally. To achieve end-to-end security, policies need to be attached to data (i.e. "sticky policies" [9]). A survey and taxonomy of enforcement of sticky policies through distributed systems is provided in [10. In contrast, our work is a contribution regarding the use of a high-level policy language with a view to translation into distributed, low-level enforcement with security labels.

Information flow control (IFC) originated in the military domain in the setting of Multi-Level Security (MLS) systems, and in that context used a limited number of centrally-defined security labels. Declassification of information was dealt with outside of the model. Myers and Liskov [4] extended IFC to decentralised enforcement allowing unprivileged principals to define and share labels and privilege over those new labels dynamically. More recently OS-level DIFC proposals 23111 protect OS processes and resources by using dynamic labels that can be created at runtime. DEFC [7] brings tag-based security to event processing systems, by allowing the labelling of event parts and assigning labels to processing components.

In the past, decentralised IFC has mainly been applied to processes within a single machine. An exception is DStar [12, which automates translation between tags in remote enforcement engines. However, DStar aims to scale to a limited number of machines, e.g. multi-tiered web applications. In contrast, the focus of our work are large-scale distributed applications that contain engines under control of independent administrative domains.

Creating a policy language for decentralised IFC has been explored in Asbestos [13]. They compute tag configurations from pairwise communication patterns between sets of processes. In contrast, DPL supports policies independently authored by multiple policy administrators in the context of multi-domain distributed applications and explicitly addresses policy compatibility checking, policy authorisation and distributed enforcement.

\section{Conclusions}

Our research is motivated with reference to use cases in complex, multi-domain scenarios found in electronic healthcare and financial services. We have presented DEFCon-Policy, a middleware that achieves end-to-end enforcement of distributed event flow control based on high-level policy. The benefits of strict, mandatory access control are coupled with the expressiveness and independence 
required by policy specification within multi-domain, distributed systems. We provide details of DPL, our event flow policy language, and sketch its formal semantics. We detail the way in which event flow policies are compiled down to be enforced using a distributed event flow control model. The evaluation of our prototype demonstrates that in both single node and distributed cases, an acceptably low overhead is incurred, while benefitting from the end-to-end, event-based security features.

In future work, we want to explore the interaction of programming languages and flow-based policy enforcement. By integrating flow constraints with programming paradigms, we can make it more natural for programmers to remain compliant with flow constraints. In addition, we want to determine the potential for interconnection of our policy and enforcement systems with existing parameterised, role-based access control infrastructures. Finally, we will acquire further experience of using DEFCon-POLICY in real-world policy environments. This will allow us to judge better the proportion of common policy requirements that are covered by DEFCON-POLICY.

\section{Acknowledgements}

This work was supported by grants EP/F042469 and EP/F044216 ("SmartFlow: Extendable Event-Based Middleware") from the UK Engineering and Physical Sciences Research Council (EPSRC).

\section{References}

1. Luckham, D.: The Power of Events: An Introduction to Complex Event Processing in Distributed Enterprise Systems. Addison-Wesley, Reading (2002)

2. Efstathopoulos, P., Krohn, M., VanDeBogart, S., et al.: Labels and event processes in the Asbestos Operating System. In: SOSP 2005, pp. 17-30. ACM, New York (2005)

3. Zeldovich, N., Kohler, E., et al.: Making information flow explicit in HiStar. In: OSDI 2006, Berkeley, CA, USA, pp. 263-278 (2006)

4. Myers, A., Liskov, B.: Protecting privacy using the decentralized label model. ACM Transactions on Software Engineering and Methodology 9(4), 410-442 (2000)

5. Chong, S., Vikram, K., Myers, A.: SIF: Enforcing confidentiality and integrity in web applications. In: USENIX Security Symposium, Berkeley, CA, pp. 1-16 (2007)

6. Papagiannis, I., Migliavacca, M., Eyers, D.M., Shand, B., Bacon, J., Pietzuch, P.: Enforcing user privacy in web applications using Erlang. In: Web 2.0 Security and Privacy (W2SP), Oakland, CA, USA. IEEE, Los Alamitos (2010)

7. Miglivacca, M., Papagiannis, I., Eyers, D., Shand, B., Bacon, J., Pietzuch, P.: High-performance event processing with information security. In: USENIX Annual Technical Conference, Boston, MA, USA, pp. 1-15 (2010)

8. Bandara, A., Kakas, A., Lupu, E., Russo, A.: Using argumentation logic for firewall policy specification and analysis. In: Distributed Systems: Operations and Management (DSOM), Dublin, Ireland, pp. 185-196 (2006) 
9. Mont, M.C., Pearson, S., Bramhall, P.: Towards accountable management of identity and privacy: Sticky policies and enforceable tracing services. In: Mařík, V., Štěpánková, O., Retschitzegger, W. (eds.) DEXA 2003. LNCS, vol. 2736, pp. 377382. Springer, Heidelberg (2003)

10. Chadwick, D.W., Lievens, S.F.: Enforcing "sticky" security policies throughout a distributed application. In: Middleware Security (MidSec), pp. 1-6. ACM, New York (2008)

11. Krohn, M., Yip, A., Brodsky, M., et al.: Information flow control for standard OS abstractions. In: SOSP 2007, pp. 321-334. ACM, New York (2007)

12. Zeldovich, N., Boyd-Wickizer, S., Mazières, D.: Securing distributed systems with information flow control. In: NSDI 2008, Berkeley, CA, USA, pp. 293-308 (2008)

13. Efstathopoulos, P., Kohler, E.: Manageable fine-grained information flow. In: EuroSys European Conference on Computer Systems, pp. 301-313. ACM, New York (2008) 